\title{
Performance Comparison of Hartley Transform with Hartley Wavelet and Hybrid Hartley Wavelet Transforms for Image Data Compression
}

\author{
H.B. Kekre, Tanuja Sarode, Prachi Natu \\ Sr. Professor, MPSTME Computer Engg. Department NMIMS University \\ hbkekre@yahoo.com \\ Associate Professor Computer Engg. Department TSEC \\ tanuja_0123@yahoo.com \\ prachi.natu@yahoo.com
}

Assistant Professor and PhD Research Scholar, Computer Engg. Department, MPSTME NMIMS University

\begin{abstract}
This paper proposes image compression using Hybrid Hartley wavelet transform. The paper compares the results of Hybrid Hartley wavelet transform with that of orthogonal Hartley transform and Hartley Wavelet Transform. Hartley wavelet is generated from Hartley transform and Hybrid Hartley wavelet is generated from Hartley transform combined with other orthogonal transform which contributes to local features of an image. RMSE values are calculated by varying local component transform in hybrid Hartley wavelet transform and changing the size of it. Sizes of local component transform is varied as $\mathrm{N}=8,16,32$, 64. Experiments are performed on twenty sample color images of size $256 \times 256 \times 3$. Performance of Hartley Transform, Hartley Wavelet transform and Hybrid Hartley wavelet Transform is compared in terms of compression ratio and bit rate. Performance of Hartley wavelet is 35 to $37 \%$ better than that of Hartley transform whereas performance of hybrid Hartley wavelet is still improved than Hartley wavelet transform by 15 to $20 \%$. Hartley-DCT pair gives best results among all Hybrid Hartley Transforms. Using hybrid wavelet maximum compression ratio up to 32 is obtained with acceptable quality of reconstructed image.
\end{abstract}

\section{Keywords}

Hartley Transform; Wavelet Transform; Hybrid Wavelet Transform; Image Compression

\section{Academic Discipline and Sub-Disciplines}

Computer Engineering-Image Processing

\section{SUBJECT CLASSIFICATION}

Transform based Image Compression

\section{TYPE (METHOD/APPROACH)}

Research Paper

\section{Council for Innovative Research}

Peer Review Research Publishing System

Journal: INTERNATIONAL JOURNAL OF COMPUTERS \& TECHNOLOGY

Vol 12, No. 6

editor@cirworld.com

www.cirworld.com, www.ijctonline.com 


\section{INTRODUCTION}

Transmission of uncompressed multimedia data like audio, video and still images requires large amount of memory and more time. Though there are advancements in processor speed, communication technologies and storage density in terms of hardware, there is a dire need of increasing data storage capacity and transmission bandwidth as todays communication is very much data intense. Compression of data provides an efficient way to increase storage capacity and reduce transmission time. The idea behind the compression is to reduce the number of bits required to represent the image. Compression is classified in two ways: lossless compression and lossy compression. In lossless compression reconstructed image is identical to original image. Hence it is used in medical image compression, text data compression etc. Methods for lossless image compression include Huffman coding, Run length coding, Bit plane coding etc. In lossy compression reconstructed image is not exactly same as original image. Hence it is useful in applications like video conferencing, and facsimile transmission, in which certain amount of error is acceptable to get increased compression ratio [1].

Mainly transform based coding is used for lossy image compression. It is based on the fact that pixels in an image exhibit a certain level of correlation with their neighboring pixels. Transformation is a process of mapping these correlated (spatial domain) coefficients to uncorrelated (frequency domain) coefficients [2].JPEG image compression is most widely used technique for lossy image compres sion. This technique is DCT oriented. DCT separates the image into set of different frequencies. From these frequencies high frequency elements are eliminated as they represent less important contents of the image [3].Transforms other than DCT has also been us ed for image compression. In [4] DST is used to compress the image. It gives acceptable values of RMSE but they are slightly higher than one obtained by DCT. Also non sinusoidal transforms like Haar, Walsh, Slant and Kekre transform have been experimented in [4]. Walsh and Haar give advantage of less computation but error in reconstructed image is quite higher than DCT. Generally to apply DCT image is divided into blocks. But it eliminates correlation across the boundaries and hence results in blocking artifacts. This drawback can be avoided by using wavelet transforms. Larger the block, more efficient is the coding. But it requires more computational power. When smaller block size is considered, lesser image distortion is observed at the cost of coding efficiency. Hence normally $8 \times 8$ or $16 \times 16$ size blocks are used.

Wavelets provide solution to this problem. Hence in recent years researchers have focused on wavelet transforms. Basic concept of wavelet is to select an appropriate wavelet function called 'Mother Wavelet' and then perform an analysis using shifted and dilated version of mother wave. Wavelet transform gives time-frequency analysis of signal. [5].It has high energy compaction capability [6]. Wavelet transform is applied on entire image rather than a block of an image. It allows a uniform distribution of compression error across entire image. Wavelet transform provides better image quality as compared to DCT at higher compression ratio [7].

\section{RELATED WORK}

Initially Haar wavelets were emphasized. But in recent years wavelet transforms of Walsh [8], Slant [9], and Kekre transforms [9] have also been studied and implemented. Wavelet transform of these respective transforms is generated using the procedure mentioned in [10]. Image compression using biorthogonal wavelet transform is proposed by Liu in [11]. A lifting scheme wavelet based transform with a modified entropy coding algorithm is proposed in [12]. It discusses effect of block sub-band coding on compression factor and quality of an image. Multi wavelet transform based on zero tree coefficient shuffling has been proposed in [13]. A simpler method of generating wavelet transform is presented in [10]. Wavelets of Haar, Cosine, Walsh, Sine, Slant are generated using the method proposed in [10,14] and Column, Row and Full Wavelet transform is used in image compression [15]. If image quality of reconstructed image is negotiable then Column wavelet Transform is used instead of Full Wavelet transforms to save number of computations. Also image compression using Real Fourier transform and its wavelet transform is proposed in [16]. Results showed that wavelets performed better than respective orthogonal transform.

\section{PROPOSED TECHNIQUE}

This paper proposes a hybrid Hartley Wavelet transform method for image compression. Results obtained by applying Hybrid Hartley Wavelet transform are compared with the results of Orthogonal transform applied on image. Wavelet transform of respective orthogonal transform is generated using equation

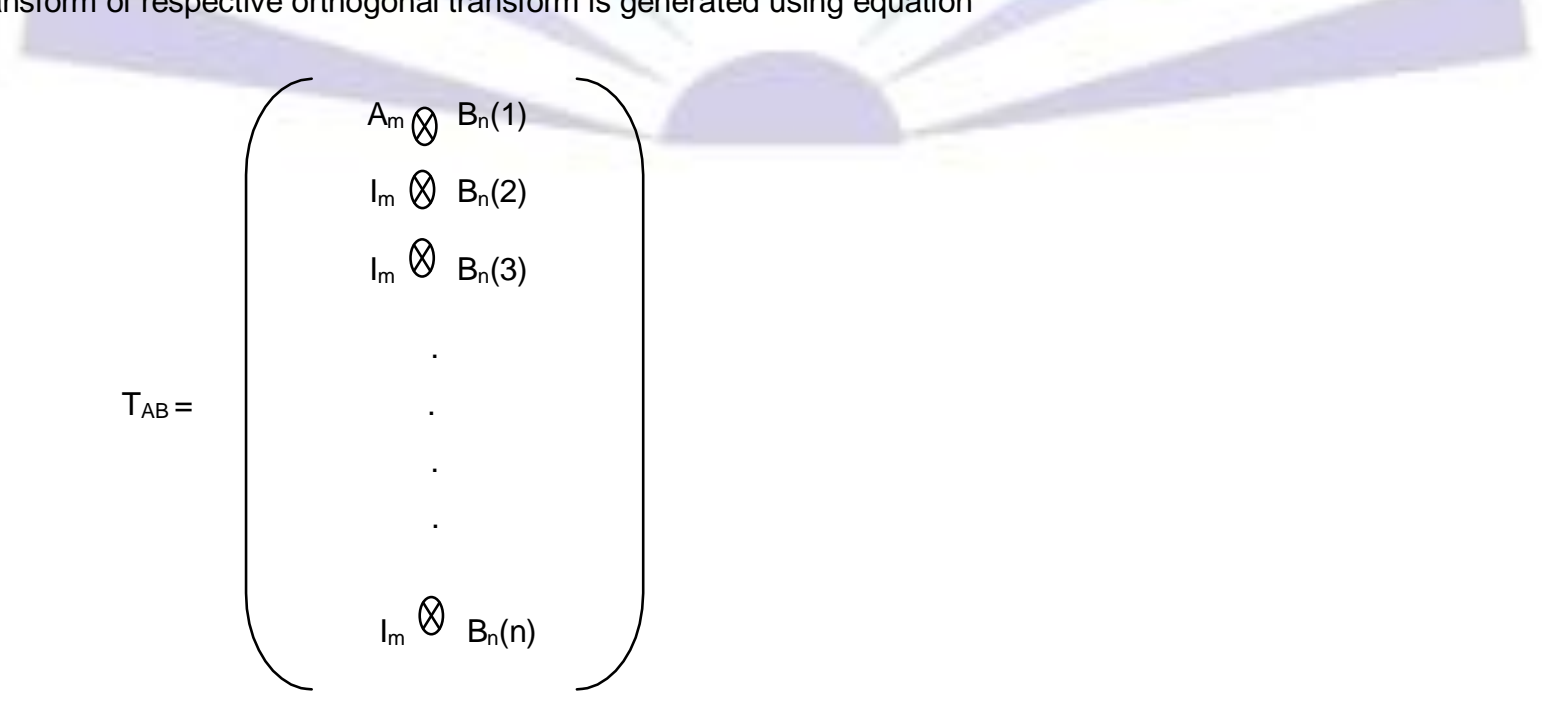


Where ' $A$ ' is of size MxM and 'B' is of size NxN and $A$ and $B$ are same orthogonal component matrices. Wavelet transform $T_{A B}$ generated will be of size MNxMN. Size of 'A' and 'B' is selected and varied as power of two and it generates $T_{A B}$ of size $M N x M N$. Hybrid wavelet transform is generated using the same kronecker product mentioned above but as it is a 'hybrid' wavelet, component transforms ' $A$ ' and ' $B$ ' are different. In hybrid Hartley Wavelet, Hartley transform is used as a base transform i.e. transform ' $A$ '. It contributes to global features of an image. Various other transforms are used as transform 'B' to contribute to local features of an image. Hybrid Hartley Wavelet transform is generated by using $8 \times 8$ base transform and $32 \times 32$ local transform. Results obtained by varying the local component transforms are compared with each other. The process is repeated by varying the size of base transform as $16 \times 16$, $32 \times 32$ and $64 \times 64$. Respective local component transform size will be 16x16, 8x8 and 4x4. Results of hybrid Hartley transform are compared with Hartley Wavelet transform and orthogonal Hartley transform. Steps to apply Orthogonal transform/ Hartley wavelet transform/ Hybrid Hartley Wavelet Transform are as follows:

1. Choose color image of size $256 \times 256 \times 3$

2. Separate Red, Green and Blue component

3. To generate Hybrid wavelet transform of image size, select Hartley Transform as base transform of size $8 \times 8$ and other orthogonal transforms like DCT/DST/Real- DFT/Walsh/DKT/Slant of size 32x32. i.e. $\mathrm{M}=8$ and $\mathrm{N}=32$

4. Generate Hybrid wavelet transform of size 256x256i.e. MN x MN.

5. Apply wavelet transform on each plane of image. Full wavelet transform of each plane is obtained as $F=[T]^{*}[f]^{*}[T] t$, where $T$ is wavelet transform matrix, $T$ is transpose of wavelet transform matrix and ' $f$ ' is individual plane of an image.

6. Sort the elements in transformed plane in descending order of their energy and eliminate lowest $256 \times 8$ elements. I.e. make them zero.

7. Apply inverse transform to reconstruct the image.

8. Calculate RMSE between original image and reconstructed image at various compression ratios.

9. Calculate rate distortion function at different bit rates.

10. Change the size of component transform to $16 \times 16$ i.e. $M=16$ and $N=16$ and repeat the steps 4 to 9 for each transform

11. Select component transform of size $32 \times 32-8 \times 8$ and $64 \times 64-4 \times 4$ and execute steps 4 to 9 again.

12. Performance of Hybrid Hartley Wavelet transforms with different orthogonal transforms is compared.

13. Also results of Hartley transform, Hartley wavelet and hybrid wavelet are compared.

\section{EXPERIMENTS AND RESULTS}

Twenty different color images are selected for experimental purpose. Each image is of size $256 \times 256 \times 3$. Experiments are performed using Matlab 7.0 on AMD dual core processor with 4 GB RAM. Images chosen are shown in Figure 1.

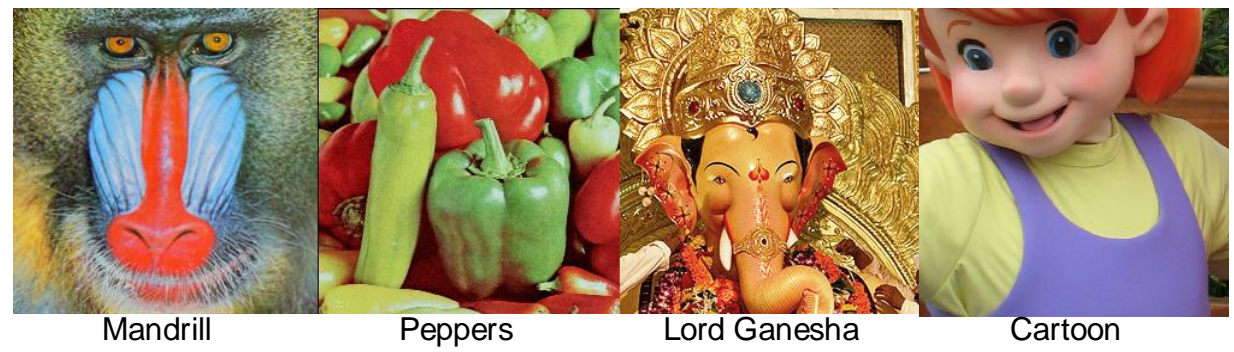




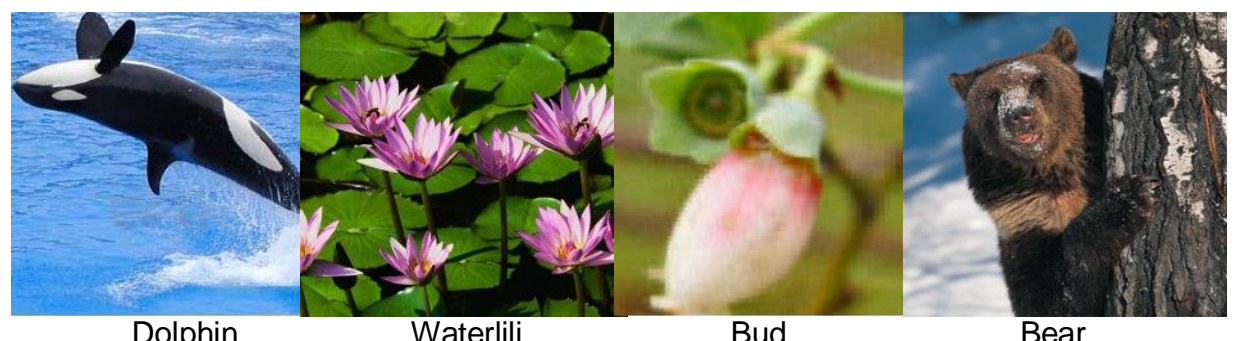

Dolphin

Waterlili

Bud

Bear

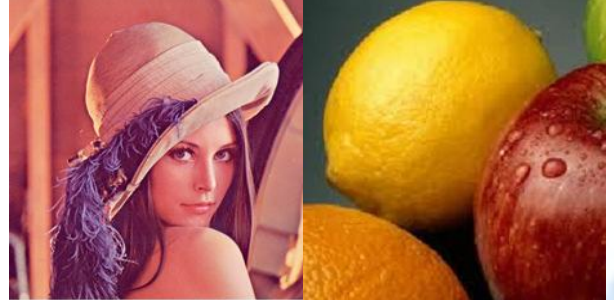

Lena

Lemon

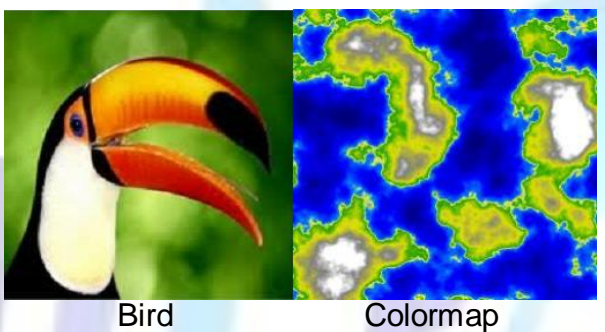

Colormap

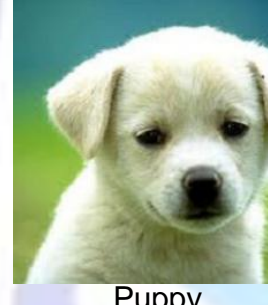

Puppy

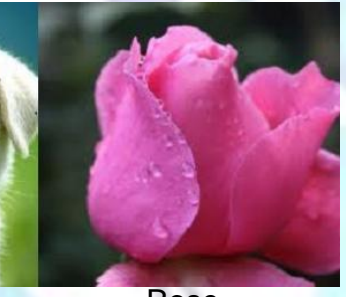

Rose

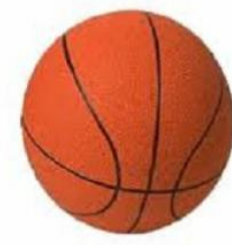

Ball

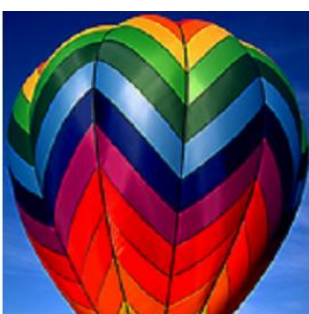

Balloon

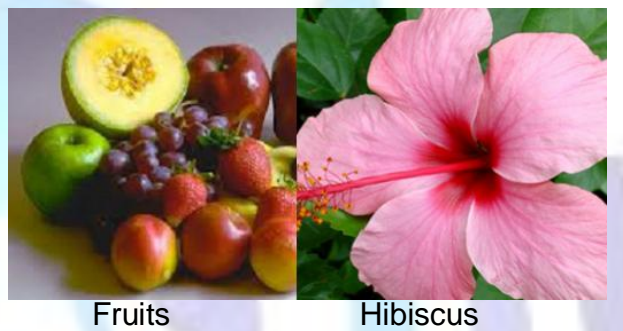

Fruits

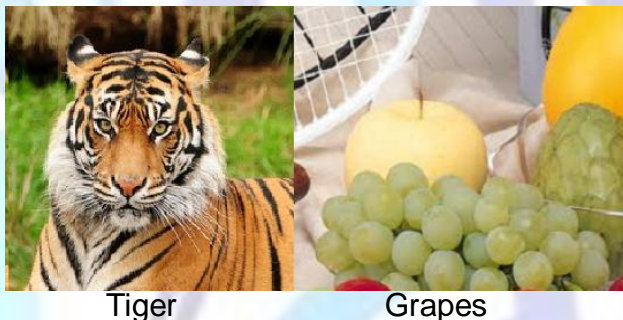

Figure1. Set of twenty test images of different classes used for experimental purpose

Figure 2 shows Average RMSE values obtained by applying different orthogonal transforms and their respective hybrid wavelet transforms with Hartley transform on images. Hartley Transform acts as a base transform and it is combined with any of the other orthogonal transform like DCT, Discrete Sine Transform (DST), Walsh, Real-DFT, Slant and Kekre Transform. For example, Hartley combined with DCT gives Hartley-DCT which is denoted as H-DCT in the graph. For all Hybrid wavelet transforms Hartley transform is of size $32 \times 32$ and local component transform is of size $8 \times 8$. From graph it has been observed that Root Mean Square Error (RMSE) obtained by applying orthogonal transform is nearly double than obtained by applying respective Hybrid Hartley Wavelet transform. In Hartley-Kekre and Hartley-Slant Hybrid Transform error is nearly ten times less than respective orthogonal Transform. H- DCT gives better performance than other hybrid wavelets as well as individual orthogonal transform. 


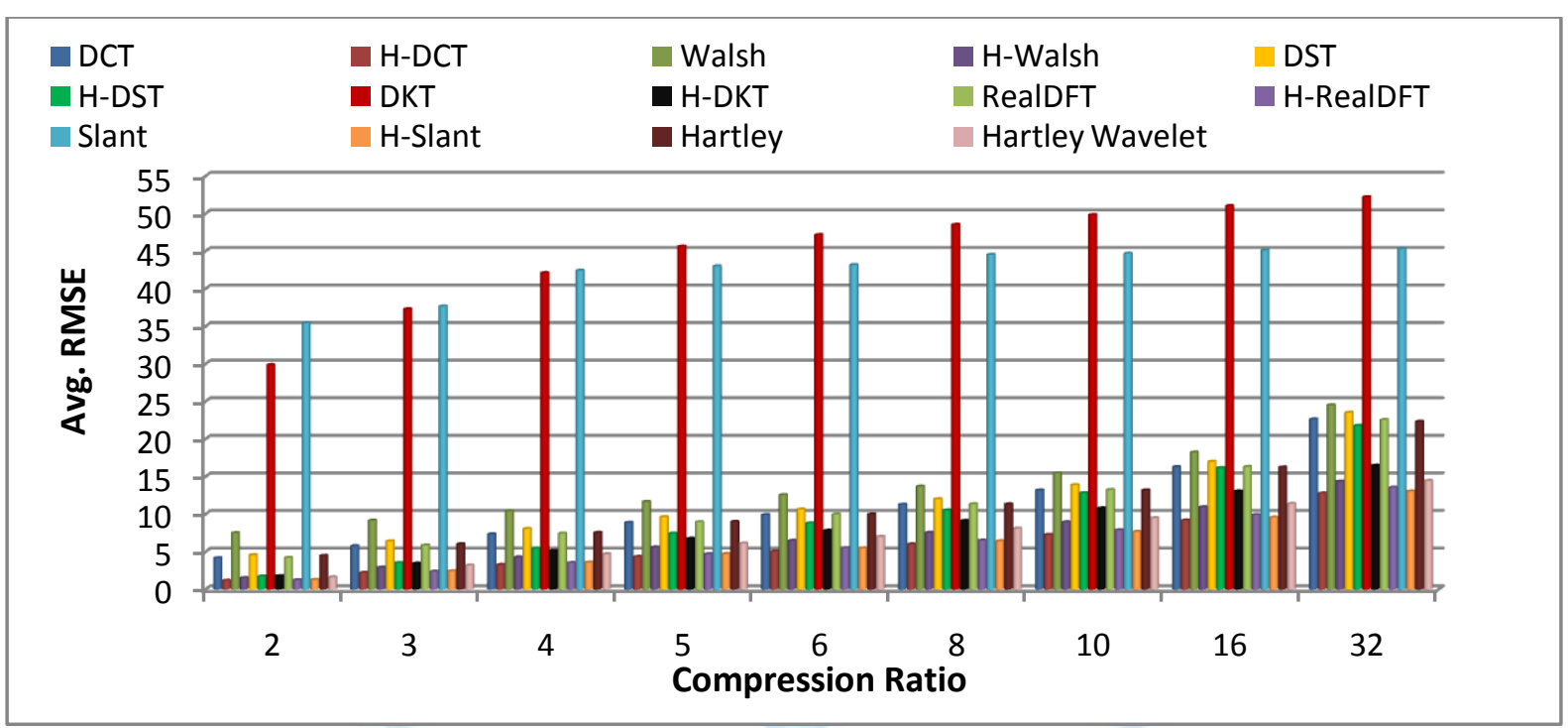

Figure 2. Comparison of Average RMSE values obtained using different orthogonal transforms and respective Hybrid Wavelet transform with 'Hartley' as a base transform of size $32 \times 32$

Figure 3 shows comparison of RMSE values for Hartley Transform, Hartley Wavelet and different Hybrid Hartley Wavelet Transforms. Error value depends upon the selection of local component transform. As DCT has higher energy compaction property, Hartley-DCT combination gives lesser value of RMSE than other combinations with Hartley transform. RMSE values up to compression ratio 32 are plotted in the graph. At this highest compression ratio HartleyDCT gives lowest value of RMSE of 12.78. Hartley-Real-DFT and Hartley-Slant give approximately similar performance. But at higher compression ratio like at 16 and 32 Hartley-Slant proves to be better. From graph it is clear that H-DCT hybrid wavelet gives twice better performance than Hartley transform.

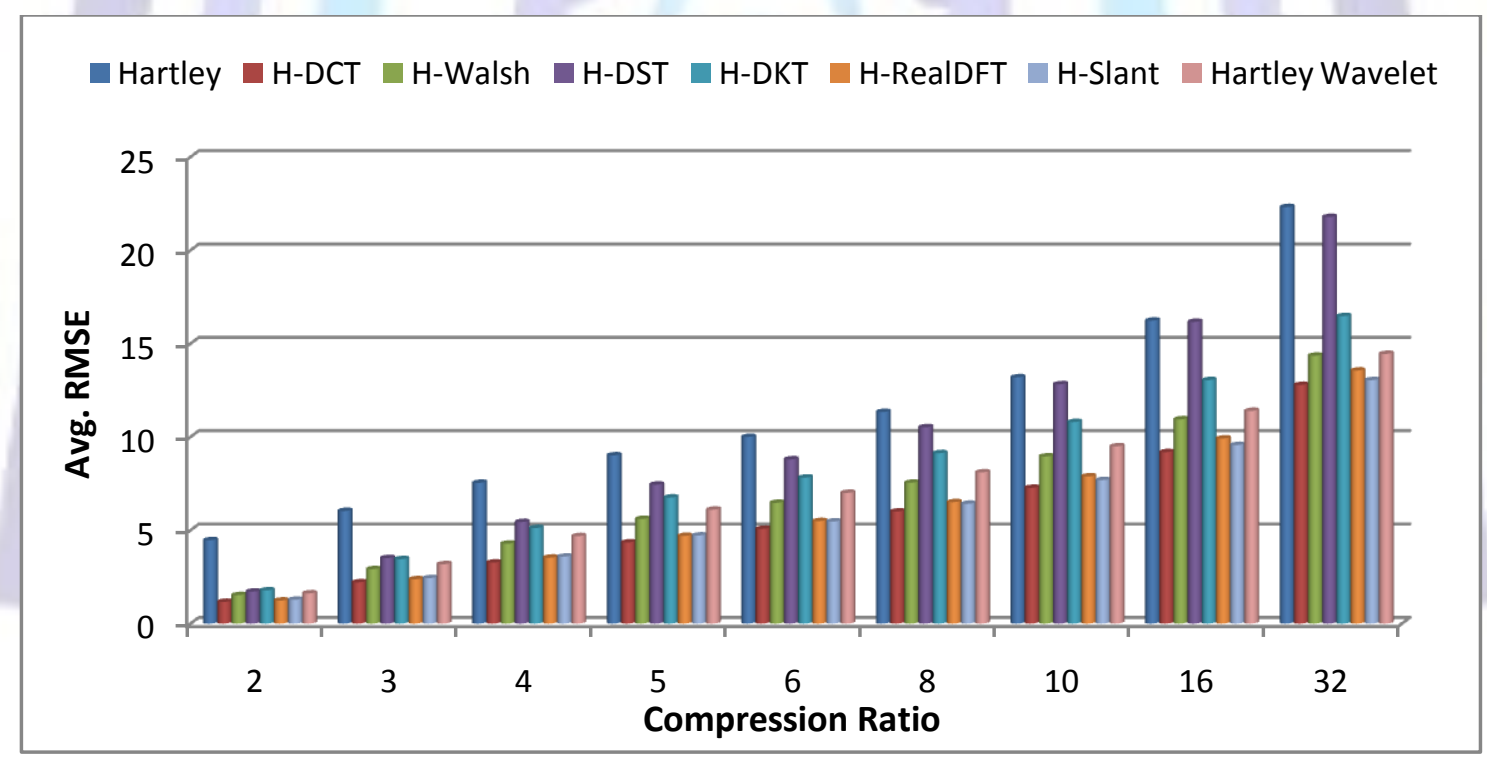

Figure 3. Comparison of RMSE values obtained by Hartley transform, Hartley Wavelet Transform and Hybrid Hartley Wavelet transform

Figure 4 shows plot of Average RMSE versus Bit Rate. Bit rate is number of bits required to represent a pixel. Hence lower is the bit rate higher is the error value. Hartley-DCT hybrid wavelet gives superior performance among all other combinations. Distortion is more when plain Hartley transform is used. Use of wavelet transform reduces the error value obtained by Hartley transform approximately by 35 to $37 \%$. Further the use of Hybrid wavelet reduces the error obtained in wavelet transform by 15 to $20 \%$. Lower is the bit rate, more is the difference between error in Hartley transform and Hybrid wavelet transform. 


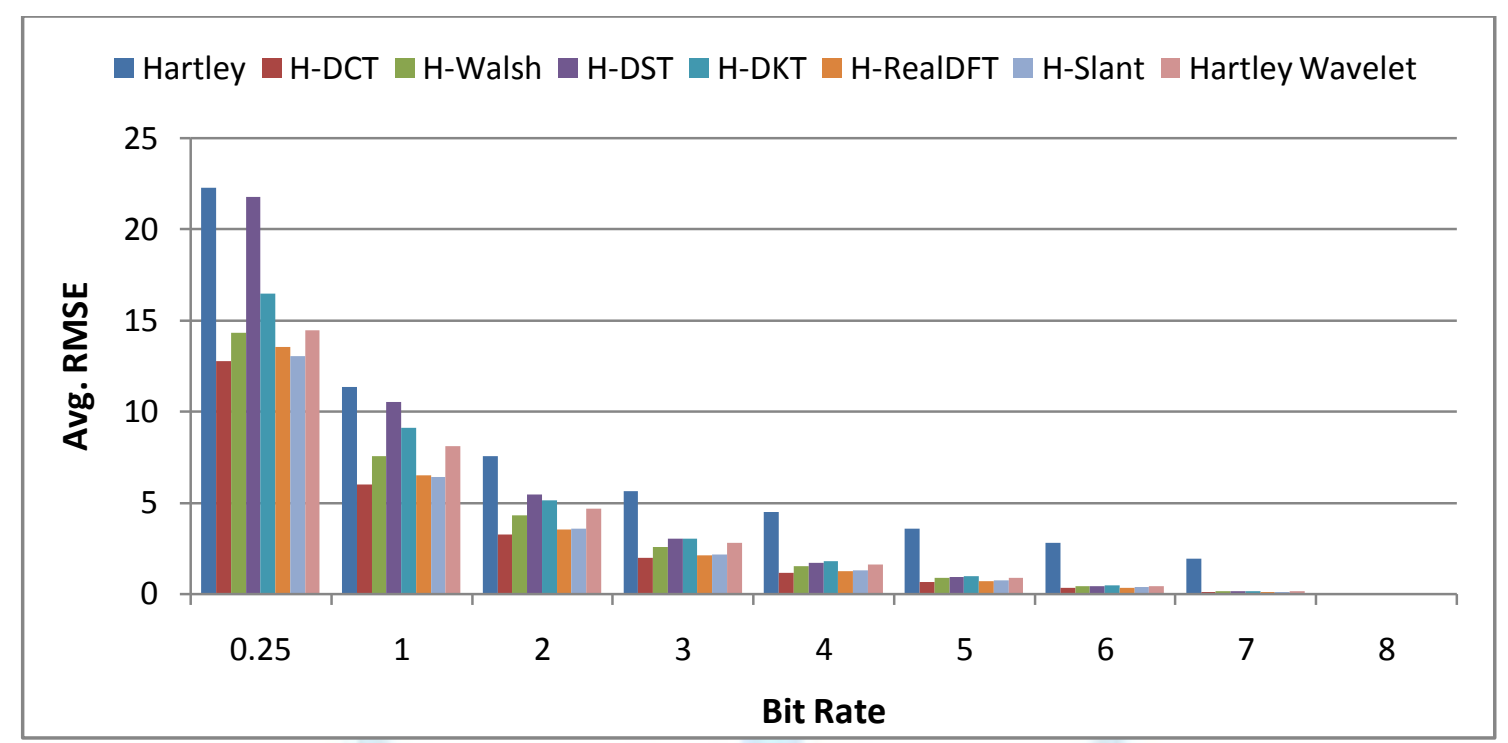

Figure 4. Error vs. Bit Rate for Hartley Transform, Hartley Wavelet Transform and Hybrid Hartley Wavelet transform

Figure 5 shows reconstructed images using different transforms mentioned in this paper. Image quality of reconstructed images is compared with original image. It has been observed that Hartley-DCT hybrid wavelet transform shows reconstructed image closer to original image. H-DST hybrid wavelet transforms shows blocking effect in reconstructed image. Slight distortion is observed due to H-DKT hybrid wavelet. For other hybrid wavelet transforms RMSE obtained is more than H-DCT hybrid wavelet, clearly indicating more distortion in the reconstructed image. For Hartley transform distortion in the image is clearly observed.
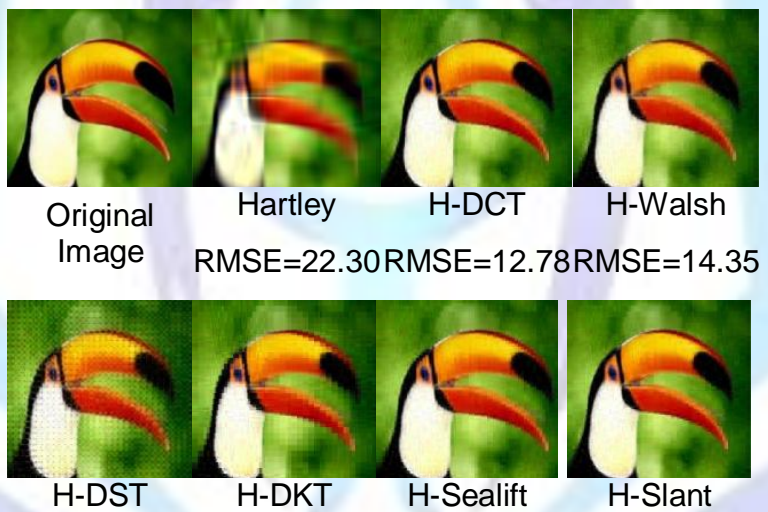

RMSE=21.7 RMSE=16.4 RMSE=13.5 RMSE=13.03

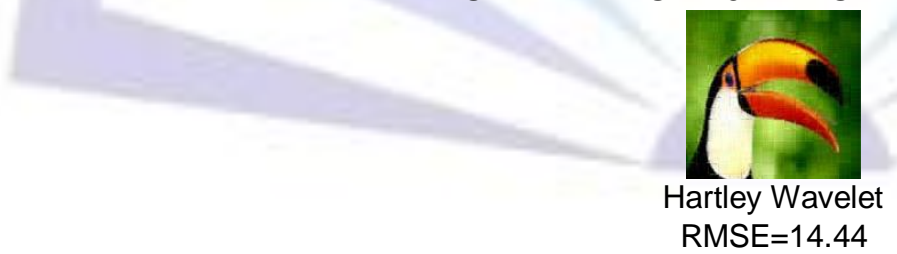

Figure 1. Reconstructed Images of 'Bird' using Hartley, Hybrid Hartley Wavelet and Hartley Wavelet Transform at Compression Ratio 32

In Figure 6 reconstructed images at compression ratio 8 are shown. As observed in the figure minimum RMSE 6.0 is obtained using Hartley-DCT hybrid wavelet. Whereas Hartley transform shows maximum error i.e.11.33.

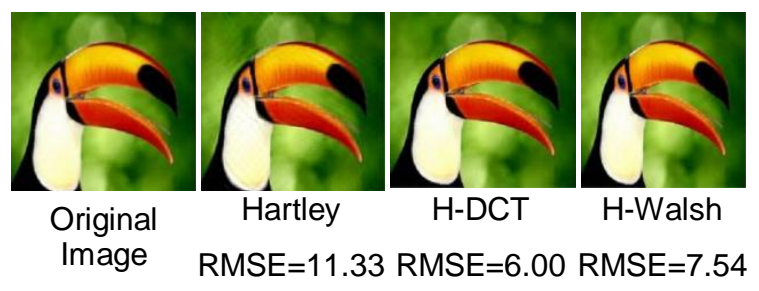




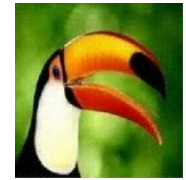

H-DST
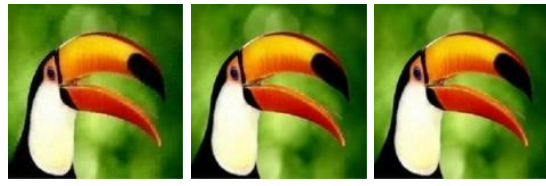

$\mathrm{H}$-DKT

H-RealDFT

H-Slant

$\mathrm{RMSE}=10.5 \mathrm{RMSE}=9.13 \mathrm{RMSE}=6.50 \mathrm{RMSE}=6.41$

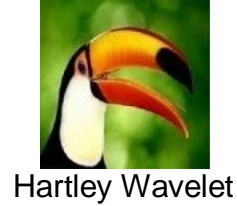

$0 \wedge C_{0} 01$

Fig.6 Reconstructed Images of 'Bird' using Hartley, Hybrid Hartley Wavelet and Hartley Wavelet Transform at Compression Ratio 8

\section{CONCLUSION}

This paper proposes image compression technique using hybrid Hartley wavelet transform. Hybrid wavelet is generated using two different component transforms. Here Hartley transform acts as a base transform and other transforms like DCT, Walsh, Slant, Kekre, Real-DFT, DST act as local transforms. Each wavelet transform gives far less error than respective orthogonal transforms and for Hybrid Wavelet Transform error is further reduced giving error two to ten times less as compared to respective orthogonal transform. Performance of hybrid Hartley Wavelet transform is compared with Hartley transform as well as Hartley wavelet transform. Hartley transform of size $32 \times 32$ and local transform of size $8 \times 8$ gives best results among all combinations of different sizes. Using hybrid wavelet maximum compression ratio up to 32 is obtained with acceptable quality of reconstructed image. Bit rate is another parameter used to observe the performance of Hartley, Hartley wavelet and Hybrid Hartley Wavelet Transform. Bit rate of 0.25 bits per pixel gives acceptable value of RMSE.

\section{REFERENCES}

[1] M. Mozammel Hoque Chowdhury, Amina Khatun, "Image Compression using Discrete Wavelet Transform", International Journal of Computer Science Issues, Vol. 9, Issue 4, No 1, July 2012, pp. 327-330.

[2] Bullmore, E., J. Fadili, V. Maxim, L. Sendur, J. Suckling, B.Whitcher, M. Brammer and M. Breakspear, "Wavelets and Functional Magnetic Resonance Imaging of the Human Brain Neurolmage", 23(1): 2004, 234-249.

[3] Anitha s "Image Compression Using Discrete Cosine Transform \& Discrete Wavelet Transform", International Journal of Scientific \& Engineering Research Volume 2, Issue 8, August 2011.

[4] H.B. Kekre, Tanuja Sarode, Prachi Natu, "Efficient Image Compression Technique using Full, Column and Row Transforms on Colour Image", International Journal of Advances in Engineering and Technology (IJAET), Vol.6 No.1. March 2013, pp. 88-100.

[5] M. Sifuzzaman1, M.R. Islam1 and M.Z. Ali, "Application of Wavelet Transform and its Advantages Compared to Fourier Transform”, Journal of Physical Sciences, Vol. 13, 2009, pp.121-134.

[6] A. S. Lewis and G. Knowles, "Image compression using the 2-D wavelet transform," IEEE Trans. Image Processing, vol. 1, pp.244-250, Apr. 1992.

[7] Z. Xiang, K. Ramchandran, M. T. Orchard, and Y. Q. Zhang, "A comparative study of DCT- and wavelet-based Image coding," IEEE Trans.Circuits Syst. Video Technol., vol. 9,Apr.1999, pp. 692-695.

[8] George Lazaridis, Maria Petrou, "Image Compression By Means of Walsh Transform", IEEE Transaction on Image Processing, Volume 15, Number 8, 2006, pp.2343-2357.

[9] H.B. Kekre, Tanuja Sarode, Prachi Natu, "Performance Comparison of Walsh Wavelet, Kekre Wavelet and Slant Wavelet Transform in Image Compression" International Journal of Advanced Research in Computer and Communication Engineering Vol. 2, Issue 10, October 2013, pp. 3834-3840.

[10] H.B. Kekre, Tanuja Sarode, Sudeep Thepade, Sonal Shroff, "Instigation of Orthogonal Wavelet Transforms using Walsh, Cosine,Hartley, Kekre Transforms and Their use in Image Compression" International Journal of Computer Science and Information Security, Vol.9, No. 6, 2011.

[11] Hong Liu, Lin Pai Zai, Ying Gao, Wen Ming Li, Jiu-Fei Zohu, "Image compressionBased on Biorthogonal Wavelet Transform", in Proc of ISCIT, 2005.

[12] Loay A. George, Aree A. Muhammad, "Intra Frame Compression Using Lifting Scheme Wavelet-Based Transformation (9/7-Tap Cdf Filter)", International Conference on Multimedia Systems And Applications, MSA 2007, June 25-28, 2007. 
[13] M. Ashok, Dr. T. Bhaskara Reddy, "Image Compression Techniques Using Modified High Quality Multi wavelets", International Journal of Advanced Computer Science and Applications, Vol. 2, No. 7, 2011.

[14] H.B. Kekre, Archana Athawale, Dipali Sadavarti, "Algorithm to Generate Kekre's Wavelet Transform from Kekre's Transform", International Journal of Engineering Science and Technology (IJEST), Vol. 2, No. 11, 2010, pp. $756-767$.

[15] H.B. Kekre, Tanuja Sarode, Prachi Natu, "Image Compression Using Column, Row and Full Wavelet Transforms Of Walsh, Cosine, Haar, Kekre, Slant and Sine and Their Comparison with Corresponding Orthogonal Transforms", International Journal of Engineering Research and Development, Volume 6, Issue 4, March 2013, pp. 102-113.

[16] H.B. Kekre, Tanuja Sarode, Prachi Natu, "Image Compression using Real Fourier Transform, It's Wavelet Transform and Hybrid Wavelet with DCT", International Journal of Advanced Computer Science and Applications,(IJACSA) Vol. 4, No.5, 2013.Pp. 41-47.

\section{AUTHOR'S BIOGRAPHY}

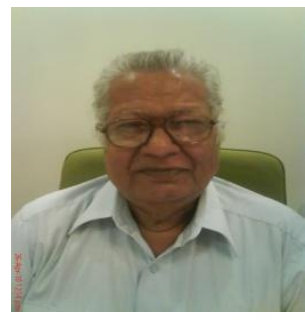

Dr. H. B. Kekre has received B.E (Hons.) in Telecomm Engineering from Jabalpur University in 1958, M.Tech (Industrial Electronics) from IIT Bombay in 1960, M.S. Engg. (Electrical Engg.) from University of Ottawa, Canada in 1965 and Ph.D. (System Identification) from IIT Bombay in 1970. He has worked as Faculty of Electrical Engg. and then HOD Computer Science and Engg. at IIT Bombay. After serving IIT for 35 years he retired in 1995. After retirement from IIT, for 13 years he was working as a professor and head in the Department of Computer Engg. and Vice Principal at Thadomal Shahani Engineering. College, Mumbai. Now he is Senior Professor at MPSTME, SVKM's NMIMS University.

He has guided 17 Ph.Ds, more than 100 M.E./M.Tech and several B.E. / B.Tech projects, while in IIT and TSEC. His areas of interest are Digital Signal processing, Image Processing and Computer Networking. He has more than 450 papers in National / International Journals and Conferences to his credit. He was Senior Member of IEEE. Presently He is Fellow of IETE, Life Member of ISTE and Senior Member of International Association of Computer Science and Information Technology (IACSIT). Recently fifteen students working under his guidance have received best paper awards. Currently eight research scholars working under his guidance have been awarded Ph. D. by NMIMS (Deemed to be University). At present eight research scholars are pursuing Ph.D. program under his guidance.

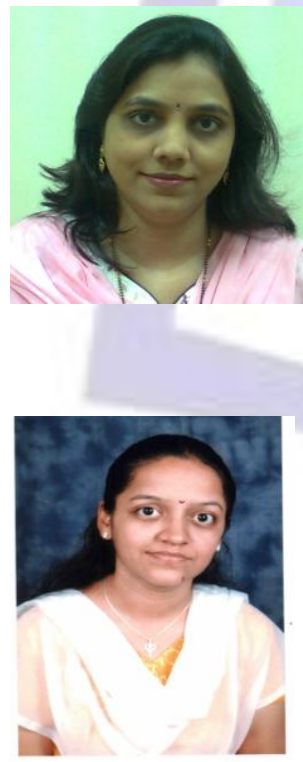

Dr. Tanuja K. Sarode has received M.E. (Computer Engineering) degree from Mumbai University in 2004, Ph.D. from Mukesh Patel School of Technology, Management and Engg. SVKM's NMIMS University, Vile-Parle (W), Mumbai, INDIA. She has more than 11 years of experience in teaching. Currently working as Assistant Professor in Dept. of Computer Engineering at Thadomal Shahani Engineering College, Mumbai. She is member of International Association of Engineers (IAENG) and International Association of Computer Science and Information Technology (IACSIT). Her areas of interest are Image Processing, Signal Processing and Computer Graphics. She has 150 papers in National /International Conferences/journal to her credit.

Ms.Prachi Natu has received B.E. (Electronics and Telecommunication) degree from Mumbai University in 2004. Currently pursuing Ph.D. from NMIMS University. She has 08 years of experience in teaching. Currently working as Assistant Professor in Department of Computer Engineering at Mukesh Patel School of Technology, Management and Engineering. Vile Parle (Mumbai). Her areas of interest are Image Processing, Database Management Systems and Operating Systems. She has 16 papers in International Conferences/journal to her credit. 